\title{
LASER-MATTER INTERACTION WITH INTENSE SHORT LASER PULSES
}

\author{
L.A. LoMpré \\ Centre d'Etudes de Saclay, DPE/SPL/SCLO, Bât. 467 \\ 91191 Gif-sur-Yvette, Cedex, France
}

Laser-matter interaction in rare gases (IIe, $\mathrm{Ne}, \mathrm{Ar}$ and $\mathrm{Xe}$ ) has been investigated using a 1 ps $\mathrm{Nd}$-glass laser pulse at $1053 \mathrm{~nm}$ with intensities between $10^{13}$ to $10^{18} \mathrm{~W} / \mathrm{cm}^{2}$. Three aspects of the interaction have been studied: the creation of multiply charged ions, the energy of the ejected electron and the propagation in an underdense plasma. At the maximum laser intensity, all electrons of the outer shell are removed, except in $\mathrm{Ne}$ for which charge states up to $7+$ are observed. Comparison of experimental data with Ammosov et al. tunneling model shows a very good agreement, indicating that ionization with a 1 ps pulse in the near infrared light mainly occurs in tunneling regime. Electrons created in a low-density medium with energies up to $5 \mathrm{keV}$ have been detected. These energies are far above the energy acquired during the ionization process, indicating that at high laser intensities electron energy is governed by ponderomotive force. Finally, the study of the propagation of an intense laser pulse in an underdense plasma shows that it is not possible to obtain simultaneously high laser intensity $\left(10^{17}-10^{18} \mathrm{~W} / \mathrm{cm}^{2}\right)$ and high electron density $\left(10^{19}-10^{20} \mathrm{~cm}^{-3}\right)$. Best conditions for solving this problem will be discussed.

PACS numbers: $32.80 . \mathrm{Fb}, 32.80$. Rm

\section{Introduction}

New short pulse laser teclinology has recently made possible the production of compact laser sources at the terawatt level. Lasers based on chirped pulse amplification in Nd-glass or Ti-sapphire materials followed by temporal compression down to, or below $1 \mathrm{ps}$, are now available in an increasing number of laboratories [1-5]. Some of these laser systems are capable of producing focused intensities of about $10^{18} \mathrm{~W} / \mathrm{cm}^{2}[6]$. Electrons oscillating in such an ultra intense laser field may become weakly relativistic. This makes possible the investigation of an entirely new class of physical effects such as relativistic self-focusing of the laser pulse in a plasma [7-11], harmonic generation of the laser frequency by relativistic electrons [12], wakefield acceleration of electrons [13], and inverse Faraday effect which is expected to produce pulsed magnetic fields of 100 tesla [14]. 
Before investigating these expected new physical effects which are related to the interaction of intense laser fields with free electrons, it is essential to have a good understanding of the plasma which results from the interaction of the ultra intense laser pulse with a gaseous target, and especially electron temperature. Electrons come from the peeling of an atom by the ultra intense laser field. As it is well known, the entire outer electronic shell of a noble gas atom can be removed. It is generally agreed that ionization can be described by multiphoton processes in the visible range, while tunnel ionization dominates in the infrared range [15]. Multiple ionization in the near-infrared is of interest because it is an intermediate regime. But it is also interesting to study what the electron energy is? The presence of a spatial intensity distribution gives rise to the so-called ponderomotive force that tends to eject the electrons from the center of the beam. Under the action of the ponderomotive force, the quiver energy of the electron in the laser field is converted into drift energy $[16,17]$. But how much of the oscillating energy is converted into kinetic energy?

Laser interaction with free electron requires high intensities because their interaction cross-sections are small [18]. As a matter of fact, they result from the interaction of free electrons with the laser field and the typical cross-sections of laser-electron interaction are several orders of magnitude smaller than those of laser-atom interaction $\left(10^{-25}\right.$ against $10^{-18} \mathrm{~cm}^{2}$ in the laser-atom interaction case). In order to observe such phenomena with a good signal-to-noise ratio, one needs to have both high laser intensities $\left(10^{18}-10^{19} \mathrm{~W} / \mathrm{cm}^{2}\right)$ and high electron densities $\left(10^{19}-10^{20} \mathrm{~cm}^{-3}\right)$. It is of interest to investigate if a terawatt laser pulse can actually be focused into a high electron-density plasma $\left(>10^{19} \mathrm{~cm}^{-3}\right)$.

The purpose of the present paper is triple: (1) give a description of multiple ionization of noble gases as a function of the peak laser intensity in the $10^{13}-10^{18} \mathrm{~W} / \mathrm{cm}^{2}$ range using a 1 ps laser pulse at $1053 \mathrm{~nm}$, (2) give an experimental value of the energy of the electron interacting with intense laser field, and (3) comment about the defocusing effects of a terawatt laser in an underdense plasma which may limit the observation of new physical effects.

\section{Experimental arrangement}

The picosecond laser used in the present experiment has been described elsewhere [2]. It is based on the chirped pulse amplification in solid state amplifiers $\left(\mathrm{Nd}^{3+}\right.$-glass) up to an energy level of $1.6 \mathrm{~J}$, followed by temporal compression down to 1 ps. A $100 \mathrm{MHz}$ CW mode-locked Nd-YLF oscillator generates a train of bandwidth-limited $50 \mathrm{ps}$ pulses at the $1053 \mathrm{~nm}$ wavelength. These pulses are injected into a $800 \mathrm{~m}$-long single-mode fiber where they are temporally stretched from $50 \mathrm{ps}$ to $250 \mathrm{ps}$ and frequency chirped from $0.2 \AA$ to $40 \AA$ by group-velocity dispersion and self-phase modulation. The pulses are further stretched to $1.5 \mathrm{~ns}$ by a pair of anti-parallel gratings. A single pulse is selected in the train and is first injected into a regenerative amplifier and then into a double-pass amplifier. The energy at the output of the double-pass amplifier is about $15 \mathrm{~mJ}$. Next, the pulse goes through a spatial filter in order to improve the spatial beam profile. The pulse energy is further amplified with three single-pass amplifiers up to a level of $1.6 \mathrm{~J}$. 
The amplified pulse is then compressed by a pair of two parallel gratings. The pulse duration is measured on every shot using a single shot autocorrelator [19]. The pulse FWIIM is found to be $1.1 \pm 0.1 \mathrm{ps}$, assuming a sech ${ }^{2}$ temporal profile which well describes the pulse shape. The signal-to-background power contrast ratio, measured using a third order correlation technique [20], is approximately $5 \times 10^{3}: 1$.

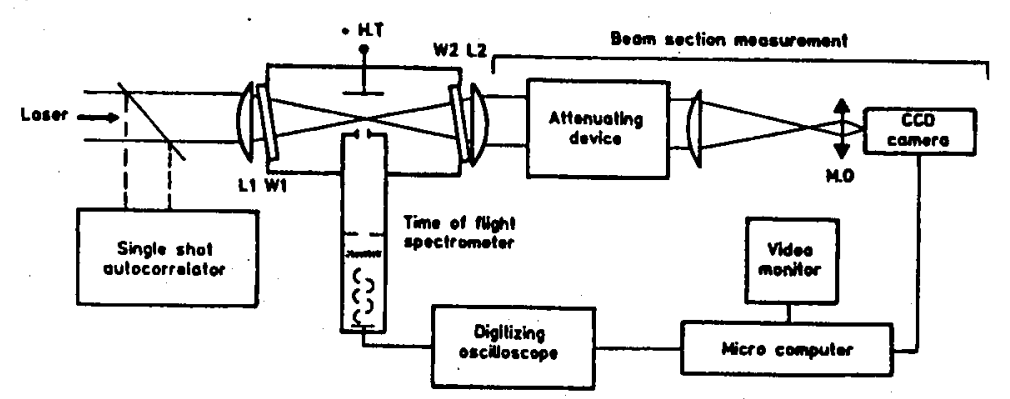

Fig. 1. General experimental set-up.

The general experimental set-up is shown in Fig. 1 [21]. The laser pulse is focused into the vacuum chamber by a $f=200 \mathrm{~mm}$ lens $\left(L_{1}\right)$. A second lens $\left(L_{2}\right)$, identical to $L_{1}$, is used to collimate the beam before measuring the focal spot size [6]. A $14 \mu \mathrm{m}$ focal spot diameter with a $15 \%$ relative uncertainty has been found. Energy measurements are performed with a pyroelectric energy meter set behind the first glass plate of the attenuating device. The error on the energy measurement is about $10 \%$ in the full energy range scanned in this experiment. Taking into account the uncertainty on focal spot size and on pulse duration measurements, the error on laser intensity measurement is within $45 \%$.

The vacuum chamber is pumped to $5 \times 10^{-9}$ torr and filled with a spectroscopically pure rare gas. A background pressure of a few $10^{-9}$ torr is required to eliminate the impurity signals from the ion spectra. For ion measurements, this problem is particularly important for high charge state ions coming from heavy atoms. Their time-of-flight can match with the one of light elements like $\mathrm{II}^{+}, \mathrm{N}^{+}$ or $\mathrm{O}^{+}$ions. The $\mathrm{Xe}^{8+}$ ion time-of-flight, for example, matches with the $\mathrm{O}^{+}$ion time-of-flight. The pressure can be varied from $10^{-6}$ to $10^{-4}$ torr to a void saturation of the detector and charge exchange interactions.

\section{Multiply charged ion production}

\subsection{Experimental resulls}

The ions produced by lascr-atom interaction are extracted from the focal volume by a transverse static electric field of $2 \mathrm{kV} / \mathrm{cm}$. The ions are analyzed by a 30-cm-long time-of-flight spectrometer and then detected with an electron multiplier which is known to allow a single ion detection. Figure 2 shows typical time-of-flight spectra in Ar, recorded at a $3 \times 10^{17} \mathrm{~W} / \mathrm{cm}^{2}$ peak laser intensity, 


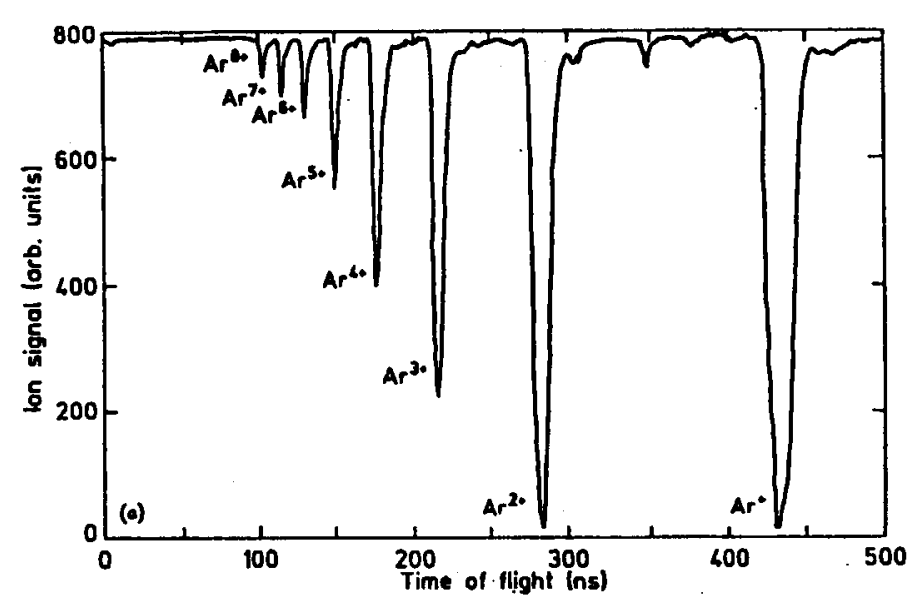

Fig. 2. Typical time-of-flight Ar spectrum recorded for a $3 \times 10^{17} \mathrm{~W} / \mathrm{cm}^{2}$ peak intensity and a $10^{-6}$ torr gas pressure.

with gas pressure of $10^{-6}$ torr. The first eight ion charge states are well separated and there is no evidence of $\mathrm{Ar}^{9+}$. For a given laser intensity, the individual ion peaks are integrated in order to obtain the number of ions detected in each charge state.

For each of the four noble gases, $\mathrm{IIe}, \mathrm{Ne}, \mathrm{Ar}$ and $\mathrm{Xe}$, the experiment consisted of measuring the number of ions detected in a given charge state as a function of the peak laser intensity. Laser energy and pulse duration have been measured for every shot and focal spot size has been measured several times for each of the four gases studied. Part of the experimental results are shown in Fig. 3 for $\mathrm{Ar}$ and $\mathrm{Ne}$. The polarization of the laser is linear. Ionization threshold intensity is defined as the intensity at which one to two ions are detected. In order to have a good signal-to-noise ratio, the pressure has been set to $10^{-4}$ torr close to the ionization threshold intensity, while it was $10^{-6}$ torr near the ion signal saturation, in order to avoid a detector saturation. Data have then been normalized to a $10^{-4}$ torr gas pressure. Each data point corresponds to a single shot. The weak dispersion of the experimental points shows the high stability of the laser used. Up to the eighth charge state has been observed in Ar and Xe gases while for Ne gas, only the seventh charge state has been detected.

Except for $\mathrm{Ar}^{+}$and $\mathrm{Xe}^{+}$ions, ion-production as a function of the peak laser intensity does not follow a $I^{N}$ variation law, where $I$ is the laser intensity and $N$ - the next integer above the ratio $E_{\mathrm{i}} / h \nu$, where $E_{\mathrm{i}}$ is the ionization energy of the atom (ion) under consideration and $\nu$ - the laser frequency. The slope of the curves is less than $N$. Deviation from a $I^{N}$ variation law increases with lighter elements. For example, a slope of $7 \pm 1$ has been measured on $\mathrm{He}^{+}$ion-production curve instead of 21 as predicted by lowest order perturbation theory. This indicates that most of the atomic (ionic) species are not ionized in perturbative regime. In terms of Keldysh adiabatic parameter, $\gamma$ [22], ionization mainly occurs with $\gamma$ less 

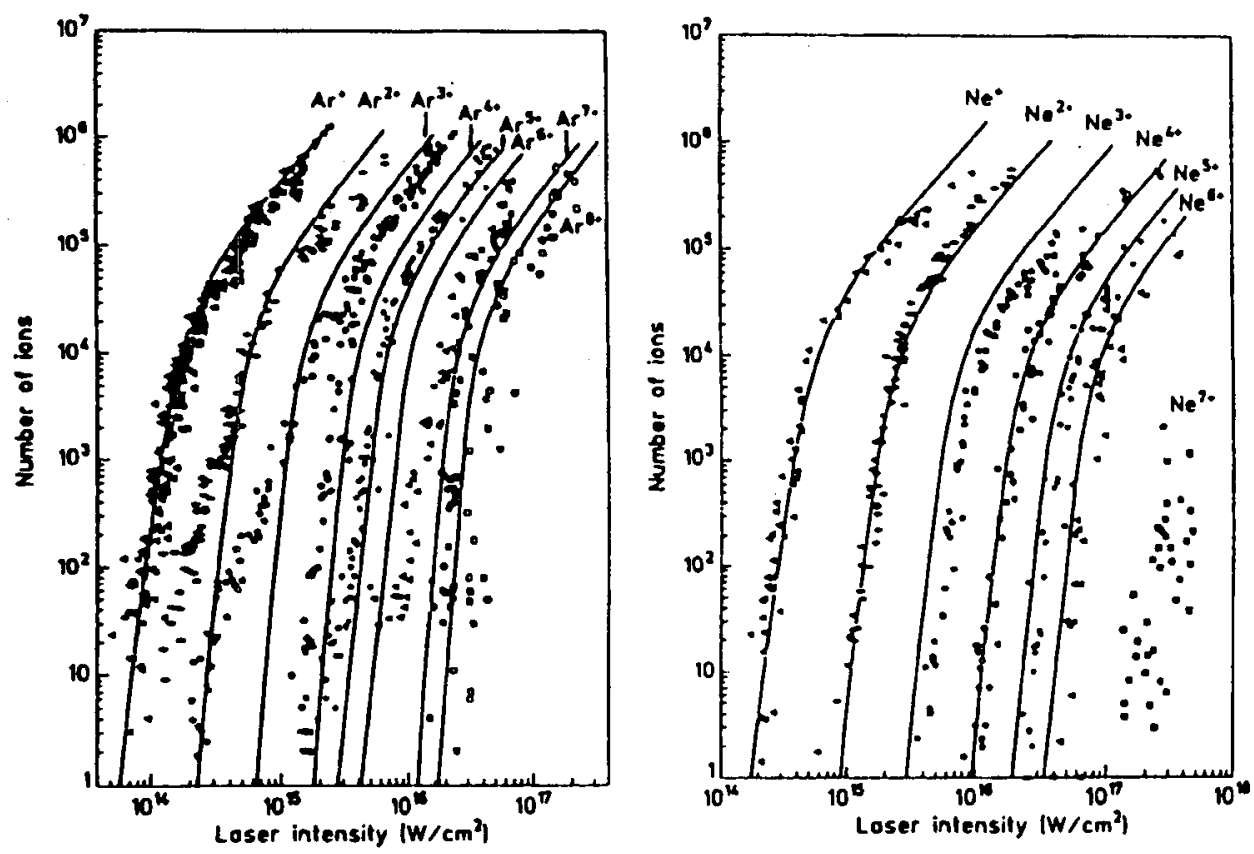

Fig. 3. Log-log plot of the absolute number of Ar and Ne ions produced as a function of the laser intensity. Solid lines are the ionization yield calculated by ADK-tunneling model. Laser polarization is linear.

than unity, i.e. in tunneling regime, for sequential processes, except for $\mathrm{Ar}^{+}$and $\mathrm{Xe}^{+}$ions for which $\gamma$ remains of the order of unity.

\subsection{Theoretical descriptions}

In order to verify our interpretation, we compared our experimental data with two simple models: Ammosov et al. model, based on a.c.-tunneling theory (ADK model), and Coulomb-barrier suppression ionization model (BSI).

\subsubsection{A.C.-tunneling theory}

An early version of a.c.-tunneling theory is due to Perelomov and co-workers [23]. They calculated the tunnel-ionization rate in an alternating electric field for arbitrary states of hydrogen atom. Ammosov et al. calculation [24] is an extension to complex atoms and atomic ions of Perelomov et al. work. Ammosov et al. give a general expression for the wave function of an arbitrary initial state, calculated under quantum defect approximation [25]. The initial state is then characterized by $n^{*}$ and $l^{*}$, which are respectively the effective principal quantum number and the effective quantum orbital momentum. For a given orbital momentum, $l$, a quantum magnetic number, $m$, and a linearly polarized electric field amplitude, $E$ (in a.u.), the tunnel-ionization rate, expressed in atomic unit, is given by 


$$
\begin{aligned}
& W_{m}(E)=\mid C_{\left.n \bullet l \cdot\right|^{2}}\left(3 E / \pi E_{0}\right)^{1 / 2} E_{\mathrm{i}} \frac{(2 l+1)(l+|m|) !}{2^{|m|}|m| !(1-|m|) !} \\
& \times\left(2 E_{0} / E\right)^{2 n^{*}-|m|-l} \exp \left(-2 E_{0} / 3 E\right),
\end{aligned}
$$

where $n^{*}=n-\delta_{l}, E_{\mathrm{i}}$ (in a.u.) is the ionization energy and $E_{0}=\left(2 E_{\mathrm{i}}\right)^{3 / 2}$ (in a.u.) the intra-atomic field strength. $\delta_{l}=n-Z\left(2 E_{\mathrm{i}}\right)^{1 / 2}$ is the quantum defect and $Z$ - the ionic charge state.

$$
\left|C_{n^{*} l^{*}}\right|^{2}=\frac{1}{2 \pi n^{*}}\left(\frac{4 \mathrm{e}^{2}}{n^{* 2}-l^{* 2}}\right)^{n^{*}}\left(\frac{n^{*}-l^{*}}{n^{*}+l^{*}}\right)^{l^{*}+1 / 2},
$$

where $\ln (\mathrm{e})=1, l^{*}=n_{0}^{*}-1 . n_{0}^{*}$ is the effective quantum number of the ground state. The $\left(3 E / \pi E_{0}\right)^{1 / 2}$ term results from averaging over one period of the laser field while the $\left(2 E_{0} / E\right)^{2 n^{*}}$ factor accounts for the long range of Coulomb potential.

Only the atomic (ionic) ground state has been considered in our ionization rate calculations. The total ionization rate is obtained by summing over the quantum magnetic number, $m$. In fact, since $E_{0}>E$, the $m=0$ term is substantially higher than others and can only be retained in the calculation. As is well known, quantum defect approximation gives a rather poor description of ground state atoms even for heavy atoms like Xe for which quantum defect approximation improvement should increase. Actually, this approximation is cxpected to be better for higher charge states and heavier elements.

As shown in Fig. 3, a surprisingly good agreement has been found for most of the ion species. Theoretical ion-production curves are obtained by integrating coupled rate equations for each ion charge state. The spatial and temporal laser intensity distributions are accounted for in our calculation. A squared hyperbolic secant temporal profile and a Gaussian spatial distribution have been assumed. Only sequential ionization processes are taken into account. It has to be emphasized that theoretical curves fit experimental data without any intensity shift. The discrepancy between theoretical and experimental curves observed on $\mathrm{Ar}^{2+}$ and $\mathrm{Ar}^{3+}$ ions, for linearly polarized light, could be attributed to direct ionization processes, i.e. $\mathrm{A}^{n+} \rightarrow \mathrm{A}^{(n+2)+}+2 \mathrm{e}^{-}$. We clearly see that the saturation of $\mathrm{Ar}^{+}$ions and $\mathrm{Ar}^{2+}$ ions produced by direct processes occurs at the same intensity level, indicating that the two species come from the same parent atom. The observation of such ionization processes in which two electrons are simultaneously removed has already been reported [26]. Here, ionization by direct processes still occurs in the multiphoton regime since the adiabatic parameter, $\gamma$, is higher than unity while $\mathrm{Ar}^{2+}$ ions created by sequential processes are produced in the tunneling regime.

The good agreement between Ammosov et al. model and our experimental results leads to the conclusion that (1) ionization should mainly occur in tunneling regime for both linear and circular polarization (not described in the present paper but discussed in Ref. [21]) and (2) ionization yields are quite insensitive to the details of the atomic structure. On this last point, one has to note that, experimentally speaking, some information may be lost due to the integration over the spatial and temporal distribution of the focused laser beam. 


\subsubsection{Barrier-suppression ionization model}

BSI model has been first introduced by Augst et al. [27]. It simply considers the effective one-dimensional potential resulting from the superposition of the Coulomb potential and a quasi-static electric field. By equating the maximum value of the effective potential to the ionization energy of the atom (ion) under consideration, one can derive the critical clectric field, $E_{\mathrm{BSI}}$ (in a.u.), which is required for the bound electron to escape without tunneling

$$
E_{\mathrm{BSI}}=\frac{E_{\mathrm{i}}^{2}}{4 Z} \text {. }
$$

The corresponding laser intensity, $I_{\mathrm{BSI}}$ (in a.u.), is given by

$$
I_{\mathrm{BSI}}=\frac{E_{\mathrm{i}}^{4}}{16 Z^{2}} \text {. }
$$

In practical units, $I_{\mathrm{BSI}}\left[\mathrm{W} / \mathrm{cm}^{2}\right]=4.00 \times 10^{9}\left(E_{\mathrm{i}}^{4}[\mathrm{eV}] / Z^{2}\right)$, where $E_{\mathrm{i}}$ is the ionization potential of the atom (ion) and $Z$ - the ionic charge state.

Since the ionization threshold intensities only depend on the electric field amplitude in BSI model, ionization threshold obtained for a circularly polarized wave is twice the one obtained for a linearly polarized field. Note that this model does not give an ionization rate. Ionization is expected to occur almost instantaneously, compared to the laser frequency, i.e. on a time scale of the order of the atomic time scale. Thus, the ionization probability is set equal to unity when $I$ is greater or equal to $I_{\mathrm{BSI}}$ and zero otherwise. The important weakness of the model is that it cannot describe the influence of the laser pulse duration which plays an important role in ionization processes. In Fig. 4, we have plotted the experimental

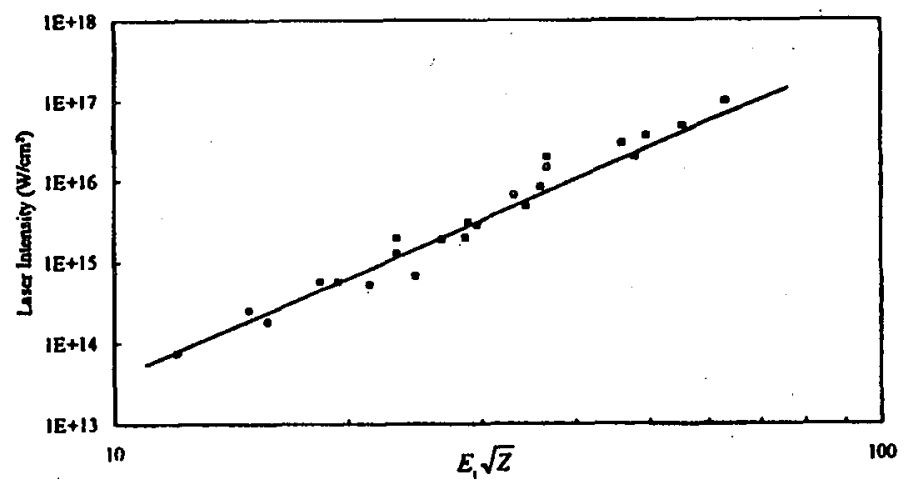

Fig. 4. Comparison between experimental ionization threshold intensities obtained in linear polarization and those predicted by. BSI model (solid line).

ionization threshold intensities, as defined previously, for linear polarization, and those given by BSI model. Only threshold intensities for ions created by sequential processes have been reported. Quite a good agreement is found only for the higher charge states and heavier gases, i.e. for $\mathrm{Ne}^{4+}, \mathrm{Ne}^{5+}, \mathrm{Ne}^{6+}, \mathrm{Ne}^{7+}, \mathrm{Ar}^{6+}$, 
$\mathrm{Ar}^{7+}, \mathrm{Ar}^{8+}$ and $\mathrm{Xe}^{8+}$ ions. In terms of Keldysh adiabatic parameter, ionization by barrier-suppression occurs for $\gamma=0.25$.

\section{Elcctron energy measurements}

\subsection{Experimental results}

For relatively low laser intensities, in the vicinity of the saturation intensity, the electron energy spectrum has been extensively studied. It has been shown that an atom can absorb more photons than the minimum number required to ionize the atom (above threshold ionization, ATI). The energy spectrum of the photoclectron exhibits series of peaks spaced by the energy of one photon [28-30]. In the low frequency limit (tunneling ionization regime) the peak structure disappears when the laser intensity increases and a broad energy spectrum is observed [31]. For these experiments at very high laser intensity, it is not possible to use a high resolution electron spectrometer. A simple retarding potential has been used.

The experimental set-up is mainly the same that described previously (Fig. 1). Only the ion detection system has been replaced by an electron energy analyzer device. The vacuum chamber is pumped down to $10^{-9}$ torr and filled with spectroscopically pure xenon gas at a pressure below $10^{-5}$ torr in order to avoid space charge effects. A $100 \mu \mathrm{m}$ diameter hole has been set $10 \mathrm{~mm}$ away from the laser axis in the laser polarization direction. The diameter hole is smaller than the confocal parameter $(b=300 \mu \mathrm{m})$, defined as twice the length over which the focal section increases by a factor of 2 . The electrons which will be collected will therefore experience the same beam profile. The electron energy is then analyzed by a retarding potential analyser which is set after the $100 \mu \mathrm{m}$ collecting hole. The voltage applied to the retarding grid is varied from 0 to $10 \mathrm{kV}$, and the electrons with energy in excess of the retarding potential are collected by a secondary electron multiplier connected to a digital oscilloscope and a microcomputer. The laser energy and the pulse duration are measured for each laser shot. Each data point results from the average over ten shots. The uncertainty in the signal is better than $10 \%$.

Figure 5 shows a retarding potential curve obtained at $2 \times 10^{17} \mathrm{~W} / \mathrm{cm}^{2}$. The experiment has been performed using a $100 \mu \mathrm{m}$ diameter hole in order to analyse only the energy of the electrons coming from the maximum laser intensity region of the interaction volume. The electron number starts to decrease from $400 \mathrm{~V}$ and a significant amount is detected up to $5 \mathrm{kV}$. More than $13 \%$ of the electrons have energy in excess of $3.5 \mathrm{keV}$. Each retarding potential curve is differentiated to obtain an energy spectrum and the average value of the energy is deduced. The average energy is shown in Fig. 6 as a function of the laser intensity. A comparison is made with the maximum energy at the laser peak intensity integrated over the $100 \mu \mathrm{m}$ length interaction volume.

At such a high laser intensity, it is well known that the final energy spectrum depends not only on the initial kinetic energy of the electrons, but on the evolution of the free electron in the laser field [32]. The presence of the spatial intensity distribution gives rise to ponderomotive force which tends to eject the 


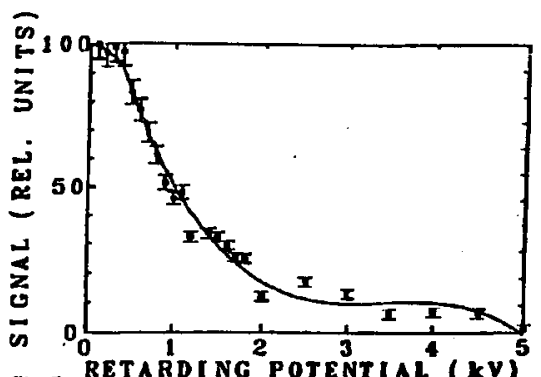
Fig. 5

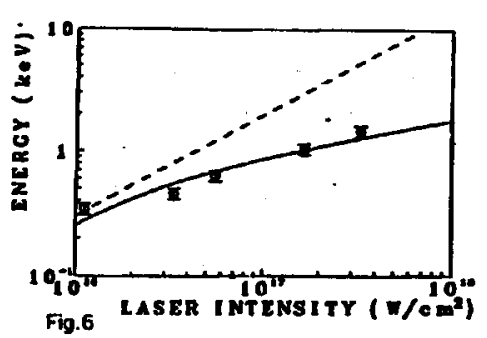

Fig. 5. Relative number of clectrons collected with energy in excess of the retarding potential as a function of the retarding potential. Solid line is a best spline fit to the data. Laser intensity is $2 \times 10^{17} \mathrm{~W} / \mathrm{cm}^{2}$. Xe pressure is $10^{-5}$ torr. The laser is linearly polarized.

Fig. 6. Log-log plot of the average value of the electron kinetic encrgy as a function of the laser intensity. Circles represent experimental data; dashed line, the integration over the focal volume of the quiver energy; full line, electron energy obtained by numerical simulation of the trajectories submitted to the ponderomotive force (see text).

electrons from the center of the beam. The maximum quiver energy $E_{\mathrm{e}}$, which can be converted into kinetic energy if the laser pulse duration is long enough, is

$$
E_{\mathrm{e}}=\frac{q^{2} F^{2}}{4 m \omega^{2}}
$$

where $F$ is the laser electric field, $\omega-$ the laser frequency, $q$ and $m$ are respectively the charge and the mass of the electron. In practical units

$$
E_{\mathrm{e}}[\mathrm{eV}]=9.33 \times 10^{-14} I\left[\mathrm{~W} / \mathrm{cm}^{2}\right] \lambda^{2}\left[\mu \mathrm{m}^{2}\right] \text {. }
$$

Taking into account the different charges states up to $Z$ the average value of the quiver energy becomes as a function of $I_{0}$ the laser intensity at the center of the beam and $I_{\mathrm{th}}^{i}$ the threshold ionization intensity for different charge states

$$
\left\langle E_{\mathrm{e}}\right\rangle=9.33 \times 10^{-14} \lambda^{2} \sum_{i=1, Z}\left(I_{0}-I_{\mathrm{th}}^{i}\right) / \sum_{i=1, Z} \ln \left(I_{0} / I_{\mathrm{th}}^{i}\right) .
$$

The overestimated value observed in Fig. 6 (dashed line) is due to the spatio-temporal laser field distribution. The maximum energy that electron will acquire will depend on its position in the laser beam with respect to the center of the laser beam. In fact, the electron will never see the maximum laser intensity. They may have left the interaction volume by ponderomotive force energy conversion before the electrons see the maximum laser intensity.

Numerical simulations have been performed taking into account the laser intensity field spatio-temporal distribution and treating the ionization dynamics via the ADK tunneling model as described previously. Electron trajectories are calculated using a Runge-Kutta routine in a spatially Gaussian beam and a square sine temporal profile. The average energies obtained by calculation are shown in 
Fig. 6 (full line). A good agrcement with experimental data is found and the difference never exceeds the $10 \%$ uncertainty on the experimental values of the electron energy.

These results show clearly that the electron energy spectrum coming from ionization of a gas in an intense laser field is dominated by the evolution of the free electron in the laser field. The conversion of quiver into kinetic (or driven) energy being done by the ponderomotive force.

\section{Defocusing effects in an underdense plasma}

\subsection{Experimental results}

The experimental set-up is basically similar to the one described earlier (Fig. 1) $[34,35]$. The ion detection system has been replaced by an optical transmission relay system which makes an image of the emilted zone on a CCD camera, in order to monitor the fluorescence. The observation is made perpendicularly to the laser axis. The signal received by the CCD camera is digitized and memorized. The interaction chamber is filled with a spectroscopically pure noble gas at a pressure varying from $1 \mathrm{mbar}$ to $1 \mathrm{bar}$. He and Ar have been used in this experiment. The pressure is measured with a Pirani gauge giving an accuracy of $30 \%$ in the $10^{-6}$ to 1 bar range. A small amount $(4 \%)$ of the incident laser power is sent to a photodiode in order to insure the monitoring of the laser energy.

For the transmission measurements, a calorimeter working in the $1 \mathrm{~mJ}-10 \mathrm{~J}$ range has been used to measure the output powcr. The output beam is stopped down by the $40-\mathrm{mm}$ diameter collimating lens, giving rise to a $12^{\circ}$ full detection angle. The spectral range of the observed fluorescence is limited by the CCD detector and is between $400 \mathrm{~nm}$ and $1200 \mathrm{~nm}$.

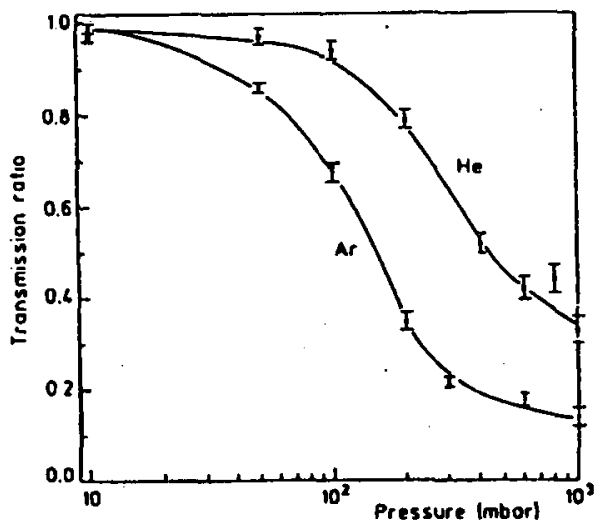

Fig. 7. Transmission ratio of a 0.1 TW laser pulse focused in a vacuum chamber filled with $\mathrm{Ar}$ or $\mathrm{He}$ at a pressure which is varied from 10 to $10^{3} \mathrm{mbar}$.

Figure 7 shows the transmission measurements as a function of the pressure for two different rare gas, Ar and Ile. The general shape of the curves is indepen- 
dent of the incident power and exhibits for low pressures, below 20 or 30 mbar, a transmission of the order of unity. The modified beam divergence is smaller than the detection angle. At intermediate pressures the transmitted power decreases rapidly. There is a net change in the beam divergence. At higher pressures, i.e. around atmospheric pressure, there is again a kind of plateau. It seems that the beam divergence becomes quite insensitive to a pressure change. The small amount of transmitted power measured at high pressure comes probably from the part of the leading edge of the pulse with an intensity below that required to ionize the medium and which consequently propagates through a neutral gas.

Figure 8 shows digitized images of the fluorescence emitted in the range of 400-1200 nm. The incident laser power is about $0.1 \mathrm{TW}$. From the top to the

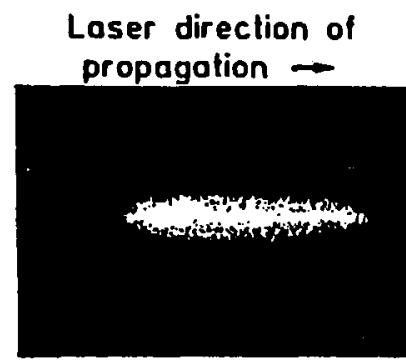

(a)

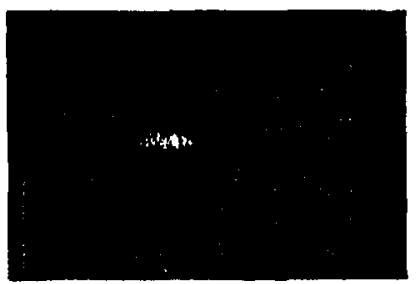

(b)

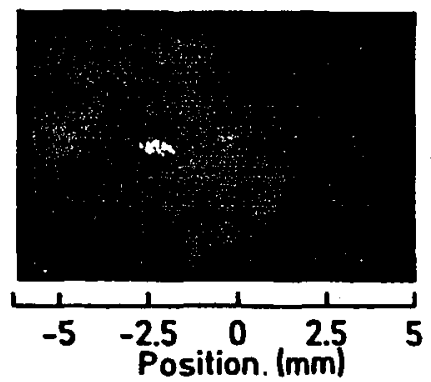

(c)

Fig. 8. Fluorescence images for different Ar pressures: (a) $10 \mathrm{mbar}$, (b) $100 \mathrm{mbar}$, and (c) $10^{3}$ mbar. The incident laser power is about $0.1 \mathrm{TW}$.

bottom, pressures are respectively $10 \mathrm{mbar}, 100 \mathrm{mbar}$ and $1 \mathrm{bar}$. The laser propagates from the left to the right of the figure. Clearly, it appears that the maximum emission of light is shifted towards the laser when the gas pressure is increased. As the emitted fluorescence is an increasing function of the laser intensity, the 
maximum emission of light coincides with the maximum laser intensity. Therefore, Fig. 8 shows that the focal point is shifted when the pressure is increased. Moreover, there is a net cliange in the emitting zone geometry: it goes from a cylindrical geometry at 10 mbar to a spherical one at 1 bar. Such a change could be interpreted as a modification of the focusing conditions if we assume that no significant change in the plasma dynamics occurs between 10 mbar and 1 bar.

The focus shifts as a function of pressure and of the incident laser power. It appears that the maximum of the fluorescence shift can be very important, larger than $5 \mathrm{~mm}$. This corresponds to more than thirty times the confocal parameter. A study of the laser focusing in vacuum and a careful measurement of the spot size have been performed at full power. The attenuating device used (Fig. 1) consists in sending the collimated output beam on four high-quality glass plates reflecting $4 \%$ and antireflection coated on the other side (or wedged). Then the beam is focused by a $f=500 \mathrm{~mm}$ lens. The image of the focusing point is analyzed by a microscope objective associated with a CCD camera. It is digitized by a video card connected to a computer. This experimental device makes it possible to measure the spot size in different planes along the propagation axis without any deformation of the wave front. It has been verified that the focusing does not change for an incident power in the 0.003-1 TW range and spot size measurements have been performed on a large scale: $\pm 10 \mathrm{~mm}$ from the bcst focus. Beam size is varying typically from $150 \mu \mathrm{m}^{2}$ at the best focus to $5 \times 10^{5} \mu \mathrm{m}^{2}$ at $10 \mathrm{~mm}$.

\subsection{Interpretation of the resulls}

Let us assume that for any gas pressure the beam focusing stays the same in vacuum and in the plasma in the focusing part before the position of the shifted focus. The shift of the focus position corresponds to an increase in the minimum spot size obtainable and consequently to a decrease in the maximum intensity. It is then possible to deduce the maximum intensity obtainable as a function of the gas pressure. The minimum spot size $S_{\min }$ is deduced from the beam scction measurements achieved under vacuum at the position corresponding to the observed shift. Figure 9 shows the maximum intensity $I_{\max }=P / S_{\min }$, where $P$ is the peak power, which can be reached in $\mathrm{He}$ and $\mathrm{Ar}$, as a function of the gas pressure. Each point reported on the curves corresponds to several incident laser powers. Any increase in the incident laser power is counterbalanced by an increase in the minimum beam section. In argon for example, for a given pressure, the same maximum intensity accessible is obtained for different laser powers. Between 10 mbar and 100 mbar, a fast decrease in $I_{\max }$ is observed, going down from a few $10^{17}$ to $10^{15} \mathrm{~W} / \mathrm{cm}^{2}$. Above $100 \mathrm{mbar}$, the decrease in $I_{\max }$ is slower, especially between 200 mbar and 1 bar where the maximum intensity obtainable remains constant and is about $5 \times 10^{14} \mathrm{~W} / \mathrm{cm}^{2}$. This value corresponds roughly to the laser intensity necessary to saturate the production of $\mathrm{Ar}^{+}$ions [21]. In the case of He atoms, this limit is much higher, typically 2 to $3 \times 10^{15} \mathrm{~W} / \mathrm{cm}^{2}$, value which also corresponds to the saturation intensity of $\mathrm{He}^{+}$ions.

Qualitative explanations of the defocusing behaviour can be given. The main part of the laser pulse travels through a plasma produced by the leading edge of 


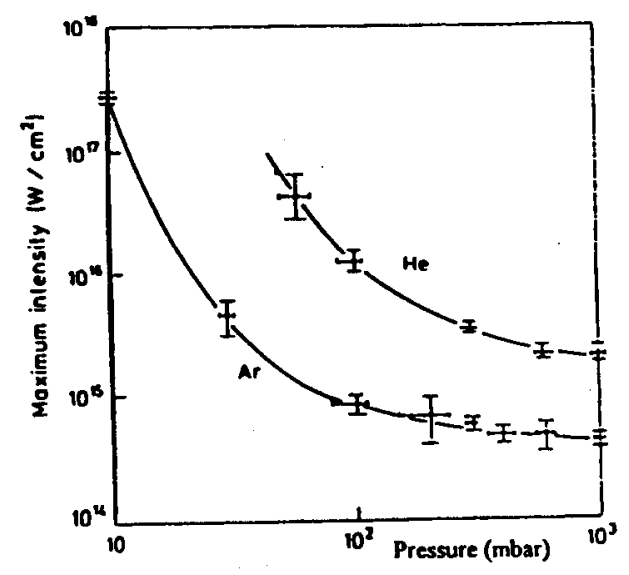

Fig. 9. Maximum laser intensity reachable as a function of $\mathrm{Ar}$ or He pressure. The asymptotic values correspond roughly to the saturation intensities of the singly-charged ion species.

the pulse. Whereas electron-atom collisions initjated by multiphoton ionization can lead to a gas breakdown in the case of a nanosecond pulse, this phenomenon does not occur in the present experiments, even at one bar, because of the short interaction time (1 ps) $[36,37]$. The threshold intensity required to obtain a gas breakdown $I_{\mathrm{br}}$, is given by

$$
I_{\mathrm{br}}=\frac{8.67 \times 10^{-10} \omega_{0}^{2} E_{\mathrm{i}}}{\sqrt{\nu_{0}} N_{\mathrm{a}} \tau_{L}}
$$

where $\omega_{0}$ is the laser frequency, $E_{\mathrm{i}}$ - the gas ionization potential, $\nu_{0}$ - the electron initial kinetic energy, $N_{\mathrm{a}}$ - the atom density and $\tau_{L}$ - the pulse duration. Threshold intensities given by this expression are in good agreement with a large set of experimental data. Under our experimental conditions, i.e. for one bar pressure of argon for example, $\omega_{0}=1.79 \times 10^{15} \mathrm{~s}^{-1}, \tau_{L}=1.1 \times 10^{-12} \mathrm{~s}, E_{\mathrm{i}}=15.755 \mathrm{eV}$ and assuming $\nu_{0}=1 \mathrm{eV}$, the threshold intensity is $1.5 \times 10^{15} \mathrm{~W} / \mathrm{cm}^{2}$, which is three times greater than the maximum intensity obtainable (Fig. 9). This explains why no breakdown is observed.

Beam propagation through the plasma is governed by the refractive index $n(r, z, t)$, which is given by

$$
n(r, z, t)=\sqrt{1-\omega_{\mathrm{p}}^{2} / \omega^{2}}=\sqrt{1-N_{\mathrm{e}}(r, z, t) / N_{\mathrm{c}}}
$$

with the plasma frequency $\omega_{\mathrm{p}}$ defined as

$$
\omega_{\mathrm{p}}^{2}=N_{\mathrm{e}}(r, z, t) e^{2} / m \varepsilon_{0}^{2}
$$

where $N_{\mathrm{e}}(r, z, t)$ is the electron density, $N_{\mathrm{c}}$ - the critical density $\left(N_{\mathrm{c}}=10^{21} \mathrm{~cm}^{-3}\right.$ for $\lambda=1053 \mathrm{~nm}), \varepsilon_{0}$ - the vacuum dielectric constant, $r$ - the radial position, $z$ - the position along the propagation axis and $t-$ the time. The electron density builds up first on laser axis at the peak of the Gaussian radial intensity profile. Here, the, plasma is produced by multiphoton ionization of the gas. This 
mechanism which is a strongly nonlinear function of the laser intensity, leads to a radial electron density profile much steeper than the laser intensity profile. As a result, the refractive index is minimum on the laser axis and the plasma acts as diverging lens for the laser beam. It should be pointed out that even at 1 bar, the index of refraction change is only $1 \%$. Nethertheless, this small change induces a dramatic defocusing eflect. Under a few assumptions the divergence coming from the refractive index can be given by [38]:

$$
\frac{D}{D_{0}}=\frac{2 \pi S_{0} \Delta n}{\lambda^{2}}
$$

where $D$ is the modified divergence, $D_{0} \longrightarrow$ the natural divergence of the Gaussian beam, $S_{0}$ - the section at the waist, $\lambda$ - the wavelength, and $\Delta n$ - the refractive index change. For $\Delta n=1 \%$, the divergence becomes ten times greater than the natural divergence of the Gaussian beam. This effect is gas dependent. Defocusing occurs at lower gas pressure in Ar than in He, because, due to the possibility to create multiply charged ions, the electronic density could be higher in Ar than in $\mathrm{He}$, for a given pressure and intensity.

\section{Conclusion}

In summary, some results on multiple ionization of four noble gases ( $\mathrm{He}, \mathrm{Ne}$, Ar and Xe) using a $1 \mathrm{ps}, 1053 \mathrm{~nm}$ laser in the $10^{13}-10^{18} \mathrm{~W} / \mathrm{cm}^{2}$ intensity range has been discussed. At the maximum laser intensity, all electrons of the outer shell are removed except for $\mathrm{Ne}$. Up to $\mathrm{Ar}^{8+}$ and $\mathrm{Xe}^{8+}$ ions has been observed while the $\mathrm{Ne}^{7+}$ ion has been detected. Comparison of experimental data with ADK tunneling model shows a very good agreement, indicating that ionization with 1 ps pulse and near infrared radiation mainly occurs in tunneling regime. Furthermore, ion curves and theoretical ones exlibit the existence of direct double ionization from $\Lambda \mathrm{r}$ and $\mathrm{Ar}^{2+}$ (same observation in $\mathrm{Xe}$ ). Experimental data obtained in linear polarization have also been compared with BSI model, showing a very good agreement with the saturation intensities experimentally measured.

These theoretical results have been used in order to understand the electron energy measurements. It has been shown that the electron energy spectrum coming from the ionization of a gas submitted to a strong laser field can be dominated by the evolution of the free electrons in the laser field. The ponderomotive force insures the conversion of the quiver energy achieved by the electron in the driving field into kinetic energy. The maximum electron energy measured reaches $5 \mathrm{keV}$ at $3 \times 10^{17} \mathrm{~W} / \mathrm{cm}^{2}$ while the average value of the electron kinetic energy varies from $300 \mathrm{eV}$ to $1.6 \mathrm{keV}$ in the $10^{16}$ to $3 \times 10^{17} \mathrm{~W} / \mathrm{cm}^{2}$ laser intensity. These energies are in good agreement with numerical simulations taking into account the spatio-temporal laser intensity distribution and the ionization dynamics described by the ADK tunneling model.

Finally, the focusing of an intense laser beam in a rare gas has been investigated as a function of the gas pressure and incident laser power. It has been shown that the beam tends to defocus as the intensity power or pressure increases. Thus high laser intensities $\left(10^{17}-10^{18} \mathrm{~W} / \mathrm{cm}^{2}\right)$ and high electron densities $\left(10^{19}-10^{20} \mathrm{~cm}^{-3}\right)$ cannot be achieved simultaneously with infrared laser radiation. 
The defocusing has been interpreted as due to the variation of the plasma refractive index. To overcome this problem which limits the possibility to study new physical effects which are expected in the $10^{18}-10^{19} \mathrm{~W} / \mathrm{cm}^{2}$ laser intensity range, two parameters have to be controlled. First, the focus shift which is induced by the presence of the electrons: the use of a pulsed gas jet may fix the electron production in the vicinity of the maximum laser intensity. Second, to avoid high electron density gradient due to multiple ionization of atoms or/and ions, the use of hydrogen gas jet is suitable. Once the $\mathrm{H}$ atom is ionized, the only electron density evolution which can occur can be generated by the ponderomotive force which tends to remove the electrons from the center of the laser beam. This may have an important consequence because if the electron density decreases locally, the index of refraction of the medium will increase (Eq. (9)): the medium will act as a converging lens. Instead of being defocused, the laser beam will be focused, conducting to a higher laser intensity. The self-focusing of the laser beam in a localized $H$ medium would be an elegant way to produce laser intensities of $10^{19}$ to $10^{20} \mathrm{~W} / \mathrm{cm}^{2}$.

I wish to thank T. Auguste, P. Monot, G. Mainfray and C. Manus for their contributions in very helpful discussions and participation to the experiments and their interpretation. I would not forget D. Fondant, M. Bougeard and E. Caprin for their technical assistance in the experiments.

\section{References}

[1] P. Maine, D. Strickland, P. Bado, M. Pessot, G. Mourou, IEEE J. Quantum Electron. $24 ; 398$ (1988).

[2] M. Ferray, L.A. Lompré, O. Gobert, A. L'Huillier, G. Mainfray, C. Manus, A. Sanchez, A.S. Gomes, Opt. Commun. 75, 278 (1990).

[3] Y.H. Chuang, D.D. Meyerhofer, S. Augst, H. Chen, J. Peatross, S. Uchida, J. Opt. Soc. Am. B 8, 1226 (1991).

[4] A. Sullivan, H. Hamster, H. Kapteyn, S. Gordon, W. White, H. Nathel, R. Blair, R. Falcone, Opt. Lett. 16, 1406 (1991).

[5] K. Yamakawa, H. Shiraga, Y. Kato, C. Barty, Opt. Lett. 16, 1593 (1991).

[6] D. Normand, M. Ferray, L.A. Lompré, O. Gobert, A. L'IIuillier, G. Mainfray, Opt. Lett. 15, 1400 (1990).

[7] C. Max, J. Arons, A. Langdon, Phys. Rev. Lett. 33, 209 (1974).

[8] G. Schmidt, W. Horton, Comments Plasma Phys. Control. Fusion 9, 85 (1985).

[9] G.Z. Sun, E. Ott, Y.C. Lee, P. Guzdar, Phys. Fluids 30, 526 (1987).

[10] D. Barnes, T. Kurki-Suonio, T. Tajima, IEEE Trans. Plasma Sci. 15, 154 (1987).

[11] A. Borisov, A. Borovskiy, V. Korobkin, A. Prokhorov, C.K. Rhodes, O. Shiryaev, Phys. Rev. Lett. 65, 1753 (1990).

[12] E. Sarachik, G. Schappert, Phys. Rev. D 1, 2738 (1970).

[13] T. Tajima, J. Dawson, Phys. Rev. Lett. 43, 267 (1979).

[14] A. Steiger, C. Woods, Phys. Rev. A 5, 1467 (1972).

[15] S.L. Chin, W. Xiong, P. Lavigne, J. Opt. Soc. Am. B 4, 853 (1987).

[16] T.W.B. Kibble, Phys. Rev. 150, 1060 (1968). 
[17] L. Jönson, J. Opt. Soc. Am. B 4, 1422 (1987).

[18] Vachaspati, Phys. Rev. 128, 664 (1962).

[19] F. Salin, P. Georges, G. Roger, A. Brun, Appl. Opt. 26, 4528 (1987).

[20] G. Albrecht, A. Antonetti, G. Mourou, Opt. Commun. 40, 59 (1981).

[21] T. Auguste, P. Monot, L.A. Lompré, G. Mainfray, C. Manus, J. Phys. B, At. Mol. Opt. Phys. 25, 4181 (1992).

[22] L.V. Keldysh, Sov. Phys. JETP 20, 1307 (1965).

[23] A.M. Perelomov, V.S. Popov, M.V. Terent'ev, Sov. Phys. JETP 23, 924 (1966).

[24] M.V. Ammosov, N.B. Delone, V.P. Kraïnov, Sov. Phys. JETP 64, 1191 (1986).

[25] M.J. Seaton, Proc. Phys. Soc. Lond. 88, 801 (1966).

[26] A. L'Huillier, L.A. Lompré, G. Mainfray, C. Manus, J. Phys. B, At. Mol. Opt. Phys. 16, 1363 (1983).

[27] S. Augst, D. Strickland, D.D. Meyerhofer, S.L. Chin, J.H. Eberly, Phys. Rev. Lett. 63, 2212 (1989).

[28] P. Monot, T. Auguste, L.A. Lompré, G. Mainfray, C. Manus, Phys. Rev. Lett. 70, 1232 (1993).

[29] P. Agostinin, F. Fabre, G. Mainfray, G. Petite, N.K. Rahman, Phys. Rev. Lett. 42, 1127 (1979).

[30] P. Kruit, J. Kimman, II.G. Muller, M.J. Van der Wiel, Phys. Rev. A 28, 248 (1983).

[31] E. Mevel, P. Breger, R. Trainham, G. Petile, P. Agostini, A. Migus, J.P. Chambaret, A. Antonetti, Phys. Rev. Lett. 70, 406 (1993).

[32] T.F. Gallagher, Phys. Rev. Lett. 61, 2304 (1988).

[33] J. Kupersztych, L.A. Lompré, G. Mainfray, C. Manus, J. Phys. B 21, L517 (1988).

[34] P. Monot, T. Auguste, L.A. Lompré, G. Mainfray, C. Manus, J. Opt. Soc. Am. B 9, 1579 (1992).

[35] T. Auguste, P. Monọt, L.A. Lompré, G. Mainfray, C. Manus, Opt. Commun. 89, 145 (1992).

[36] R.G. Meyerand, A.F. Haught, Phys. Rev. Lett. 11, 401 (1963).

[37] C. Grey Morgan, Rep. Prog. Phys. 38, 621 (1975).

[38] R. Rankin, C.E. Capjack, N.H. Burnett, P.B. Corkum, Opt. Lett. 16, 835 (1991). 